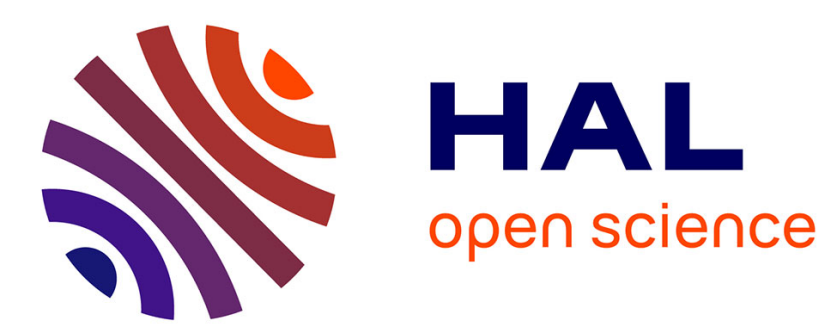

\title{
Soft-X study of buried interfaces in stratified media
}

\author{
N. Mahne, A. Giglia, Lorenzo Sponza, A. Verna, S. Nannarone
}

\section{To cite this version:}

N. Mahne, A. Giglia, Lorenzo Sponza, A. Verna, S. Nannarone. Soft-X study of buried interfaces in stratified media. Seventh International Conference on Thin Film Physics and Applications, Sep 2010, Shanghai, France. pp.79951S, 10.1117/12.888179 . hal-03411511

\section{HAL Id: hal-03411511 \\ https://hal.science/hal-03411511}

Submitted on 2 Nov 2021

HAL is a multi-disciplinary open access archive for the deposit and dissemination of scientific research documents, whether they are published or not. The documents may come from teaching and research institutions in France or abroad, or from public or private research centers.
L'archive ouverte pluridisciplinaire HAL, est destinée au dépôt et à la diffusion de documents scientifiques de niveau recherche, publiés ou non, émanant des établissements d'enseignement et de recherche français ou étrangers, des laboratoires publics ou privés. 


\title{
Soft-X study of buried interfaces in stratified media
}

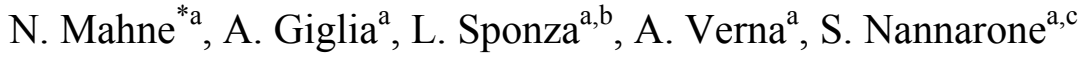 \\ ${ }^{a}$ Istituto Officina dei Materiali - CNR, Trieste (Italy); \\ ${ }^{\mathrm{b}}$ Università degli Studi di Trieste, Trieste (Italy) \\ now at Laboratoire des Solids Irradiés, École Polytechnique, Palaiseau (France) \\ ${ }^{\mathrm{c}}$ Università di Modena e Reggio Emilia, Modena (Italy)
}

\begin{abstract}
The performance of multilayer optics depends on the quality of the buried interfaces between materials, whose intermixing strongly affects their behavior. We present an experimental method to determine, in a non destructively way, the amount of material intermixing at interfaces of multilayer structures. The reflection mechanism is related to the build up in the multilayer of a standing wave field, whose peaks and the valleys move as a function both of wavelength and of incidence angle. Exploiting this fact it is possible to modulate the electric field inside the multilayer in order to have different parts of the multilayer structure excited at a different extent and in particular the buried interfaces regions. The excitation is directly proportional to the intensity of the electric field and to the concentration of a given element in the sample. The excitation can be detected with different techniques, f.i. electron core level photoemission, fluorescence, luminescence, total electron yield.

The flexibility of the experimental apparatus of the BEAR beamline (Elettra Trieste, Italy) allowed us to study some important classes of layered structures in the soft X-ray energy range, using the above mentioned techniques together with the determination of the Bragg conditions through the measurement of the specular reflectivity. We demonstrate the possibility of obtaining quantitative information on the width of the intermixing region, strongly related to the interface roughness, through the comparison with a phenomenological model of the intermixing and a numerical simulation of the standing field inside the multilayer.
\end{abstract}

Keywords: buried interfaces, multilayer.

\section{INTRODUCTION}

The performances of a multilayer mirror depends on the quality of the interfaces including sharpness, roughness and type of interface chemical compounds. The reflection mechanism is related to build up in the multilayer of a standing wave field; the positions of the peaks and valleys move as a function both of wavelength and of incidence angle [1]. Exploiting this fact it is possible to modulate the electric field inside the multilayer in order to have different parts of the multilayer structure excited at a different extent and in particular the buried interface regions.

With this in mind, the experimental setup reported in Figure 1(left) to study the buried interface between Ru and $\mathrm{Si}$ in a $\mathrm{Ru}$ capped $\left[\mathrm{Si}_{41.2} \mathrm{Mo}_{39.6}\right] \times 40$ multilayer was arranged at the BEAR beamline [2] at Elettra, by measuring the photoelectron emission from $\mathrm{Ru} 3 \mathrm{~d}$ while scanning in angle through the rocking curve in the vicinity of the Bragg condition $\left(\sim 6.1^{\circ}\right.$ grazing $)$. In this setup the reflected intensity can be simultaneously measured. Exploiting the fact that at the interface the Ru reacts with Si to form silicide [3] (presumably $\mathrm{Ru}_{2} \mathrm{Si}_{3}$ ) and that the binding energies of Ru in the silicide are different (lower in case of $\mathrm{Ru}_{2} \mathrm{Si}_{3}$ [4]), we are able to get information on the extension of the region of intermixing at the buried R-Si interface. In Figure 1 (right) the evolution of Ru 3d photoemission peak at different grazing angles is shown.

In what follows it is shown that through an appropriate modellisation of the emission of photoelectron in a multilayer as a function of incidence angle it is possible to fit the experimental data and to determine the parameters that characterize the buried $\mathrm{Ru} / \mathrm{Si}$ interface.

*mahne@tasc.infm.it

Seventh International Conference on Thin Film Physics and Applications, edited by Junhao Chu, Zhanshan Wang, Proc. of SPIE Vol. 7995, 79951S

(C) 2011 SPIE · CCC code: 0277-786X/11/\$18 - doi: 10.1117/12.888179

Proc. of SPIE Vol. 7995 79951S-1 
In section 2 the phenomenological model is presented, together with a short description of the method used for calculating the electric field and the energy transfer in the multilayer. In section 3 the experimental results are presented together with their discussion. In section 3 the conclusions are drawn.
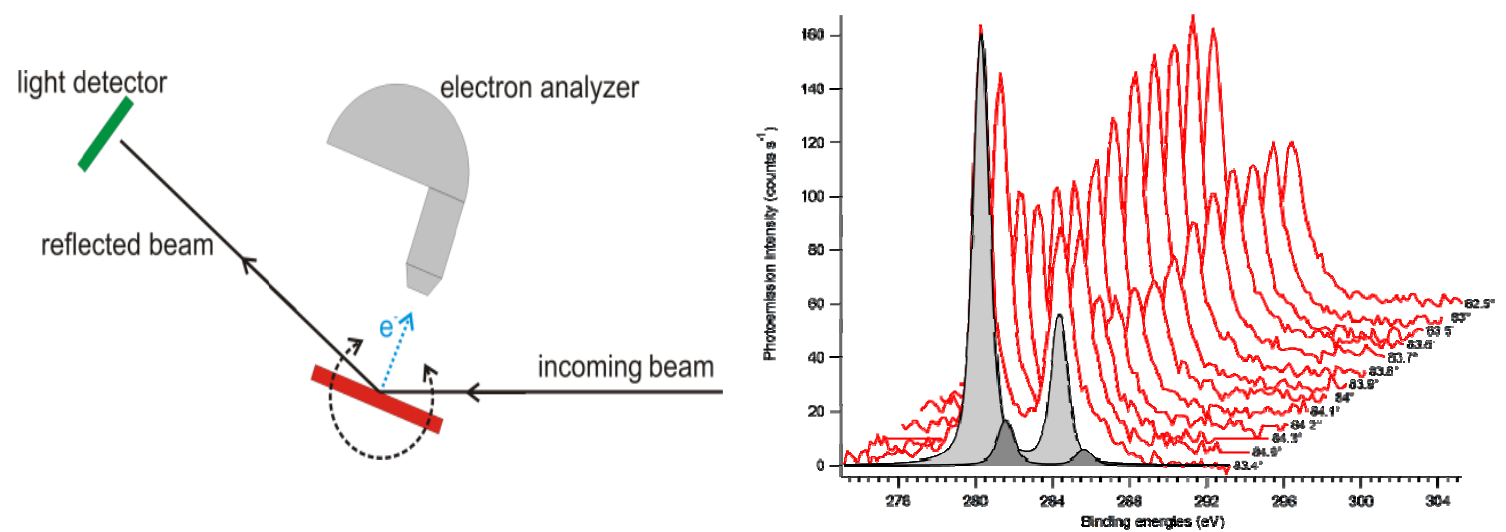

Figure 1: experimental setup (left). On the right is shown the modulation of the $\mathrm{Ru} 3 \mathrm{~d}$ photoemission signal while scanning the rocking curve around the Bragg peak. The shadowed areas are the contributions of the two different kind of Ru.

\section{PHOTOEMISSION VERSUS ROCKING ANGLE: MODEL CALCULATION}

The intensity $I_{i}\left(\omega, E_{K}\right)$ of a photoemission signal, from the $i$-th specie at a depth $z$ in the material, can be written as

$$
I_{i}\left(\omega, E_{K}\right)=\int_{-\infty}^{0} N_{i}(z) \sigma_{i} n_{i} G T\left(E_{K}\right) \lambda\left(z, E_{K}\right) \Delta n_{p h}\left(z, \omega, \theta_{i n}\right) d z
$$

where $N_{i}(z)$ is the density of atoms of the $i$-th specie, $\sigma_{I}$ is the relative photoionization cross section of the electron of specific binding energy over the sum of the cross sections of all possible excitation channels, $n_{i}$ is the occupation number of the electronic level, $T\left(E_{K}\right)$ is the transmission function of the electron analyzer at kinetic energy $E_{K}$, corrected by a geometrical factor $G, \lambda\left(z, E_{K}\right)$ is the escape depth of electrons of kinetic energy $E_{K}$ created at a distance $z$ from the surface $^{1}, \Delta n_{p h}\left(z, \omega, \theta_{i n}\right)$ is the amount of photon absorbed by the material at depth $z$, with energy $\hbar \omega$ impinging with an incidence angle $\theta_{i n}$.

The escape of the electrons is described by an escape function written as

$$
\lambda\left(z, E_{K}\right) \propto \exp \left(-\frac{|z|}{\lambda_{e}\left(E_{K}\right)}\right)
$$

where $\lambda_{e}\left(E_{K}\right)$ is the mean free path of electrons of kinetic energy $E_{K}$.

It is useful to introduce the function $\widetilde{N}_{i}(z)$ - periodic with $z$ - defined as

$$
\tilde{N}_{i}(z)=\frac{N_{i}(z)}{N_{\text {TOT }}(z)}
$$

\footnotetext{
${ }^{1} \mathrm{We}$ assume that the mean free path is independent from the nature of the material, i.e. we assume the universal curve for mean free path.
} 
where $N_{T O T}(z)$ is the total atomic density. An indicative behavior of $\tilde{N}_{i}(z)$ shown in Figure 2 for example purpose.

The number of photon absorbed per unit time in the portion of material between $z$ and $z-d z^{2}$ is proportional to the negative of the Poynting's vector:

$$
\Delta n_{p h}=\frac{\Delta E}{\hbar \omega}=\frac{-\nabla \cdot \vec{S}}{\hbar \omega}
$$

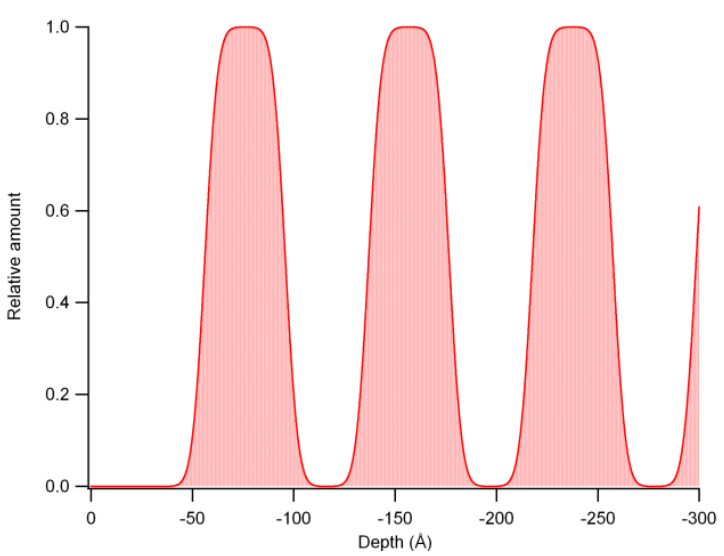

Figure 2: relative abundance $\widetilde{N}_{i}(z)$ of an element in a multilayer. The interface regions are described by $\operatorname{erf}(z ; \sigma)$ with $\sigma=5 \AA$.

We used the OPAL (Optical Properties for Anisotropic Layers) code [5] to calculate the Poynting vector of the radiation in the multilayer. This code has been developed by ourselves and, given the structure of the sample, together with the dielectric tensor of the materials for each layer, calculates the electric and magnetic field distribution in the multilayer, solving in a self-consistent way the Fresnel equation in each layer. The response of the sample illuminated by radiation of given degree of polarization is expressed through products of matrices which takes into account the boundary conditions imposed by the geometry and by the incoming electric field.

The Poynting vector is defined as

$$
\vec{S}(z)=\frac{1}{2} \sqrt{\frac{\varepsilon_{0}}{\mu_{0}}} \Re\left[\vec{E}^{*}(z) \times \vec{H}(z)\right]
$$

Since we deals only with plane waves, that is with infinite front, the divergence of the Poynting vector reduces to the $z$ derivative

$$
-\nabla \cdot \vec{S}(z)=-\frac{\partial S_{z}}{\partial z}
$$

The behavior of the Poynting vector and of its divergence can be calculated once we know the electric field $\vec{E}(z)$ and the magnetic field $\vec{H}(z)$ inside the multilayer, and it is shown in Figure 3 for the Ru capped Si/Mo multilayer at the Bragg condition $\left(\theta_{i n}=84.1^{\circ}\right)$ and out of Bragg condition $\left(\theta_{i n}=87^{\circ}\right)$.

Assuming constant both the transmission function of the analyzer and the geometrical function $G$, eq. (1) for the intensity of the photoemission signal gives

\footnotetext{
${ }^{2}$ In our modellisation we assume the axis $z$ oriented perpendicular to the surface with the positive direction above the surface of the sample.
} 


$$
I_{i}\left(\omega, E_{K}\right) \propto \int_{-\infty}^{0} \tilde{N}_{i}(z)\left(-\frac{\partial S_{z}}{\partial z}\right) \frac{1}{\hbar \omega} \exp \left(\frac{-|z|}{\lambda_{e}\left(E_{K}\right)}\right) d z
$$

In absence of analytical expressions for the function $\widetilde{N}_{i}(z)$ empirical forms are generally used [5] including the error function $\operatorname{erf}(z)$ - see also Figure 2 - defined as

$$
\operatorname{erf}(z ; \sigma)=\frac{1}{\sqrt{\pi}} \int_{-\infty}^{z} \exp ^{\prime}\left(\frac{-t^{2}}{2 \sigma^{2}}\right) d t
$$

We are interested mainly at what happens at the interfaces, where we can have an intermixing of materials and, in case, the formation of compounds.
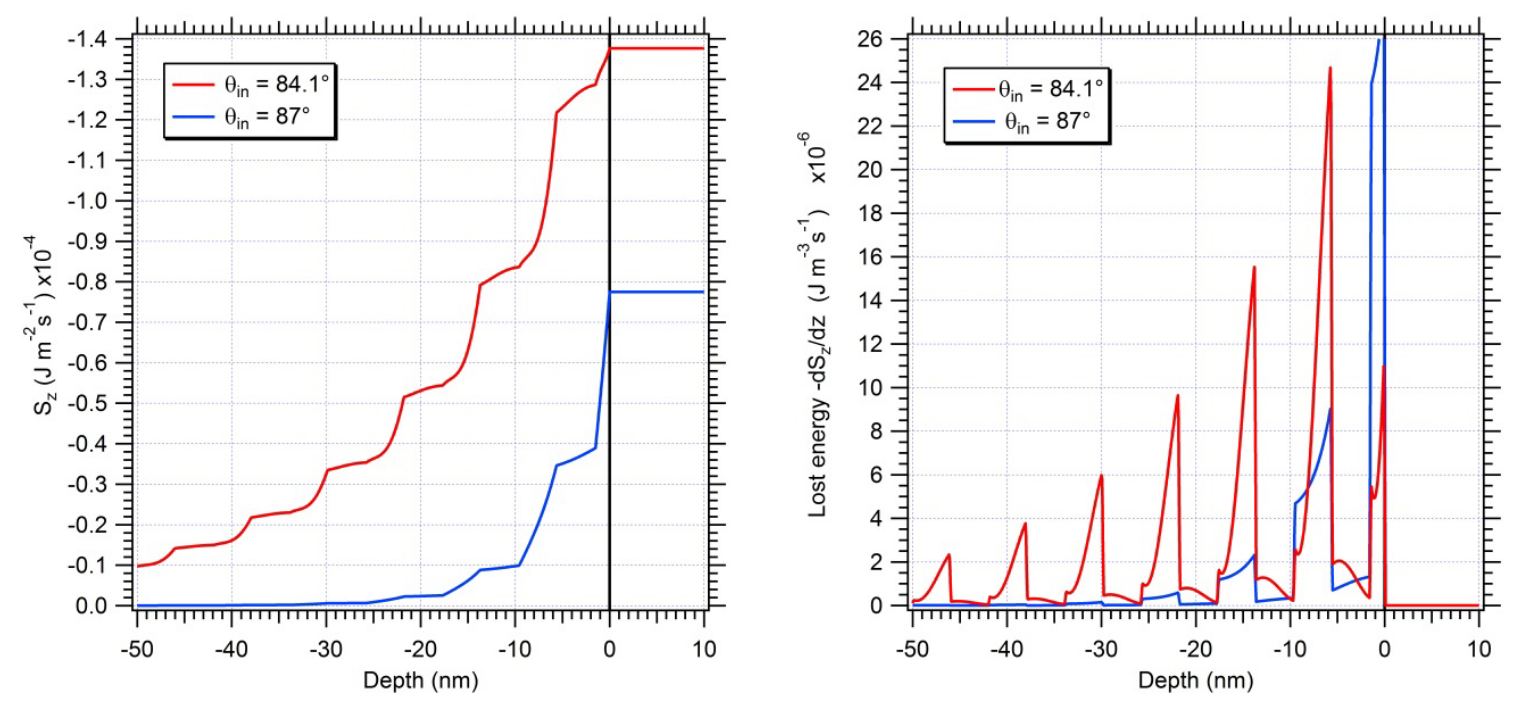

Figure 3: (left) $z$ component of the Poynting vector for the multilayer $\mathrm{Ru} /[\mathrm{Si} / \mathrm{Mo}] \times 40$ considered in this work at two different incidence angles; (right) energy absorbed by the multilayer as function of depth. 


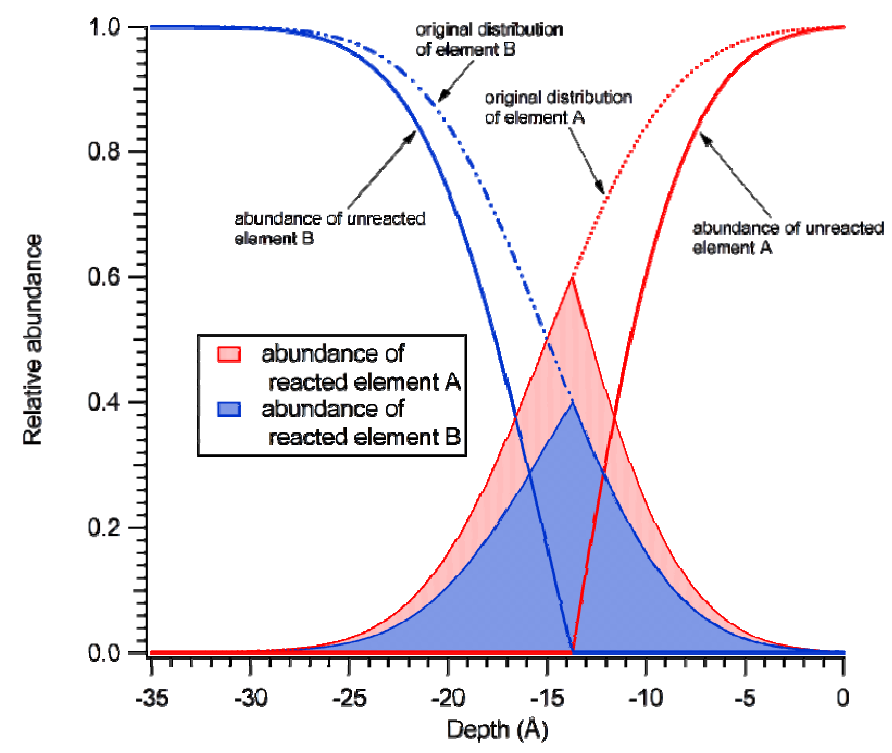

Figure 4: relative abundance of a compound $\mathrm{A}_{\mathrm{m}} \mathrm{B}_{\mathrm{n}}$ with $m=2$ and $n=3$ (like the case of $\mathrm{Ru}_{2} \mathrm{Si}_{3}$ ). The dotted lines represent the original relative abundance $\tilde{N}_{A}(z)$ and $\tilde{N}_{B}(z)$. The solid lines are the relative abundance of not reacted elements $A$ and $B$ to form the compound. The shadowed areas - resolved per element - represents the relative abundance of the compound.

The relative abundances at the interfaces reflect interface reaction, intermixing and roughness. Considering two materials $A$ and $B$ with relative abundances $\widetilde{N}_{A}(z)$ and $\widetilde{N}_{B}(z)$ (see eq. (3)), with $\widetilde{N}_{A}+\widetilde{N}_{B}=1$, it is useful to introduce the relative abundancies $\widetilde{N}_{A}^{r}$ and $\widetilde{N}_{B}^{r}$ of the reacted $A$ and $B$ materials and $\widetilde{N}_{A}^{u}$ and $\widetilde{N}_{B}^{u}$ of the unreacted materials $\left(\widetilde{N}_{A}^{r}=\widetilde{N}_{A}-\widetilde{N}_{A}^{u}\right.$, analogously for $B$ ). The unreacted abundance for $A$ (analogously for $B$ ) is given by

$$
\tilde{N}_{A}^{u}=\left\{\begin{array}{cc}
\frac{m \widetilde{N}_{A}-n \tilde{N}_{B}}{m} & \text { if } m \widetilde{N}_{A}>n \widetilde{N}_{B} \\
0 & \text { if } m \widetilde{N}_{A}<n \widetilde{N}_{B}
\end{array}\right.
$$

where $m$ and $n$ are the indexes of the brute formula of the $A_{m} B_{n}$ compound.

In Figure 4 the relative abundance of a $A_{2} B_{3}$ compound ( $\left(\mathrm{f}\right.$.i. $\mathrm{Ru}_{2} \mathrm{Si}_{3}$, as at issue here) is shown. The solid line represent the relative abundance of the not reacted atoms. The two areas indicate the relative abundance of the reacted atoms.

The function (7) can be seen as a fitting function depending - by others - on the morphology of the interfaces and on the parameter describing the roughness of the interface.

Inserting in (7) a calculated value for the $z$ component of the Poynting vector, the distribution of the excitation in the material from which the photoelectrons are emitted and the final intensity of the photoemission peak (area of the excited region) can be obtained. Results are shown in Figure 5 for the specific case of radiation of $838 \mathrm{eV}$ impinging on a multilayer $\mathrm{Ru} /[\mathrm{Si} / \mathrm{Mo}]$ with incidence angle spanning from $82^{\circ}$ to $86^{\circ}$. In these figures the photoemission peaks from $\mathrm{Ru}-$ $\mathrm{Si}$ interface, assuming the formation of $\mathrm{Ru}_{2} \mathrm{Si}_{3}$, are reported versus the rocking angle separating the contribution of $\mathrm{Ru}$ in pure metal and $\mathrm{Ru}$ in silicide. 

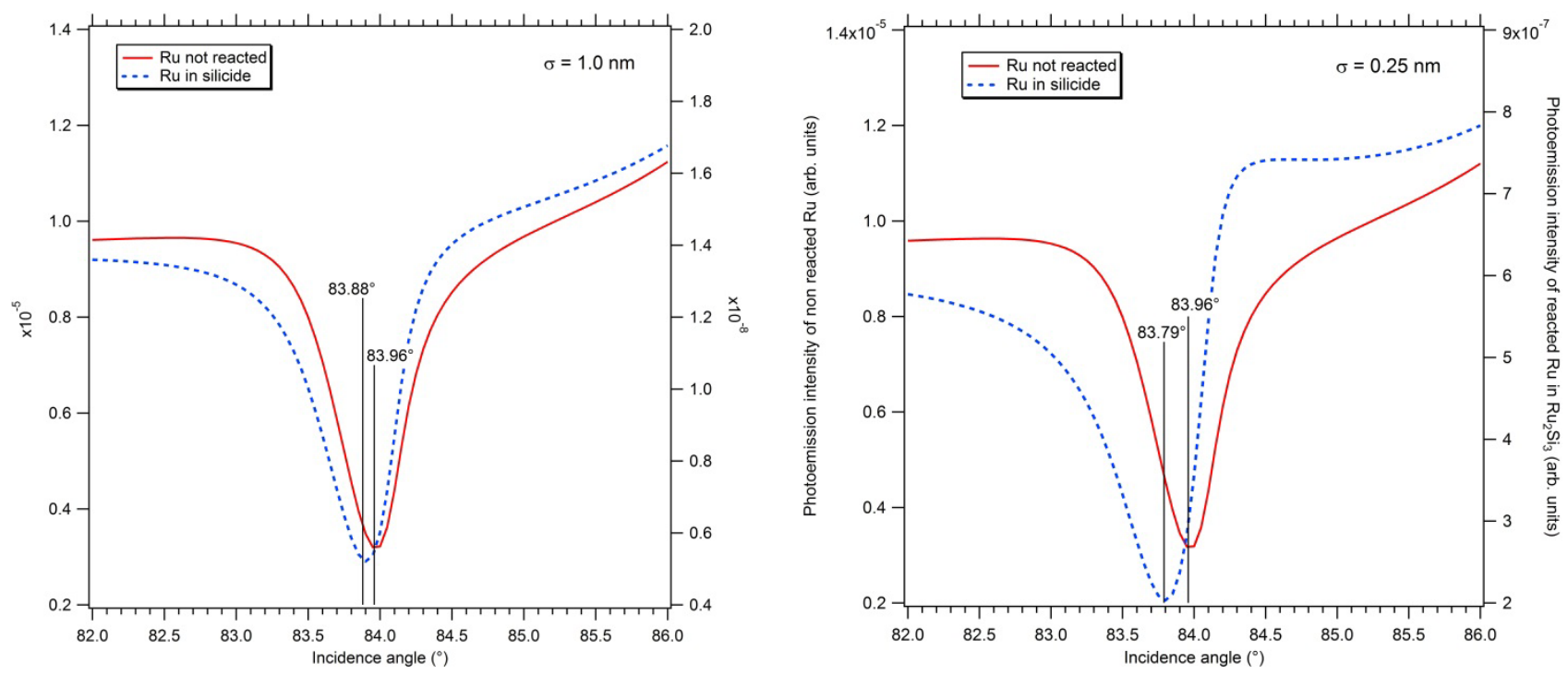

Figure 5: Calculation of the peak intensities (in arbitrary units) of the signal from "metallic" Ru and from Ru in the silicide. The calculations have been performed for two different values of $\sigma$ which characterize the interface. It is evident that there is a correlation between the distance of the two minima and the value of $\sigma$.

These results contain two important indications: (i) there is a correlation between the distance of minima: the higher the distance, the smaller the $\sigma$ of the interface; (ii) the not reacted Ru contribution - pure metal - has the minimum at lower angles. However a more sophisticated simulation code, including also detector collection and electron deviation at the interface and a more precise calculation of electric field intensity at smooth interfaces, is being developed providing the $\tilde{N}_{i}(z)$ as well as $\sigma$ as fitting functions and parameter. Results obtained for other systems are in close agreement with the above (i) and (ii) conclusions [6].

\section{EXPERIMENTAL RESULTS AND DISCUSSION}

Some measurements have been made to characterize the interface in a Si/Mo multilayer capped with $\mathrm{Ru}$. The multilayer was made by 40 periods of $\mathrm{Si}(41.2 \AA) / \mathrm{Mo}(39.6 \AA)$. A capping layer of $15 \AA$ of $\mathrm{Ru}$ was on top. The measurements have been made on the BEAR beamline at Elettra (Trieste). The particular configuration of the beamline and of the experimental chamber is particularly suited [7] for this kind of measurements.

We chose to fix the energy of the radiation illuminating the sample and to change the incidence angle of the light according to a rocking curve around the Bragg peak (see Figure 6). 


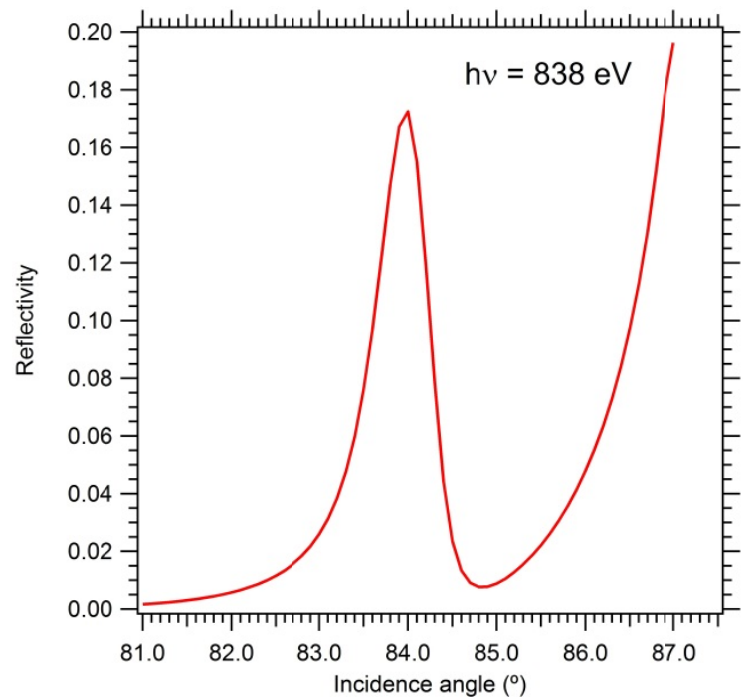

Figure 6: specular reflectivity vs. incidence angle of the multilayer after the sputtering. Photons of energy $838 \mathrm{eV}$ have been used.

Photoemission has been measured over a large range of kinetic energy (from $100 \mathrm{eV}$ to $838 \mathrm{eV}$ ), using photons of $838 \mathrm{eV}$. In order to see the deep interfaces, it was necessary to make the capping layer thinner by means of argon ion sputtering, in order to clean the sample without reducing significantly the reflectivity of the multilayer, that is without altering the structure of the electric field inside.

In this work we concentrated on the Ru 3d signal, which forms silicide when it is in contact with Si [4]. Since the Ru in the silicide has different binding energies from metallic $\mathrm{Ru}$. The best fit of the data is obtained using two Voigt doublets for two different kind of Ru (grey and red areas in the figure) and one single Voigt curve for $\mathrm{C}$ 1s peak, which has similar binding energy of $\mathrm{Ru} 3 \mathrm{~d}_{3 / 2}$. A typical photoemission lineshape is shown in Figure 7.

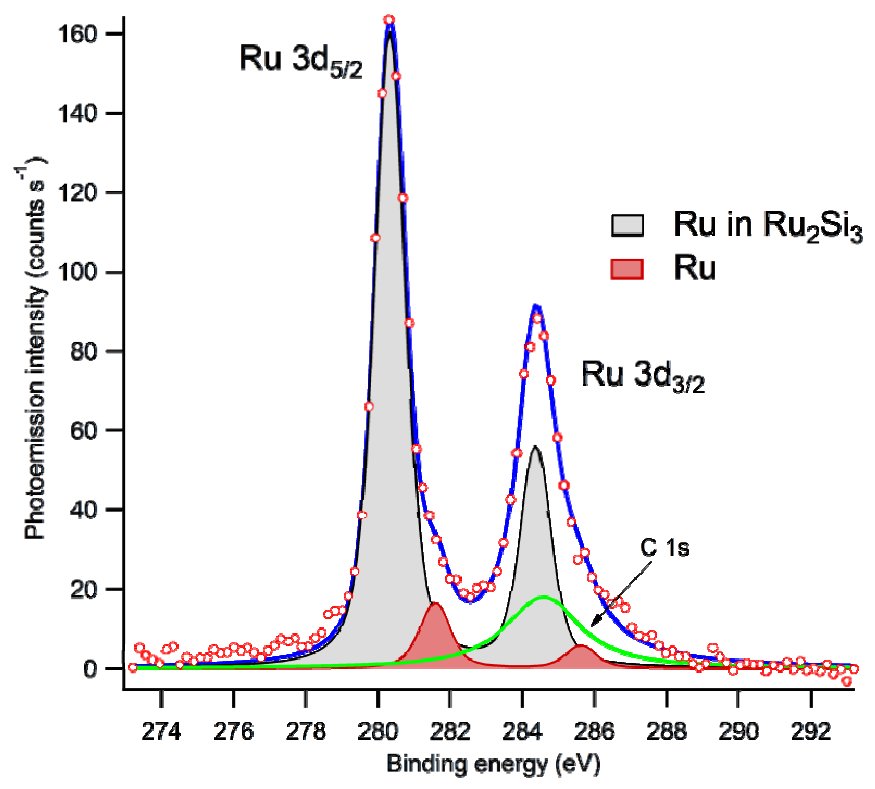

Figure 7: Ru 3d core levels. The data are well fitted using two Voiogt doublets for the $3 \mathrm{~d}$ levels and one single Voigt curve for the component of $\mathrm{C} 1 \mathrm{~s}$ which has the same binding energy. 


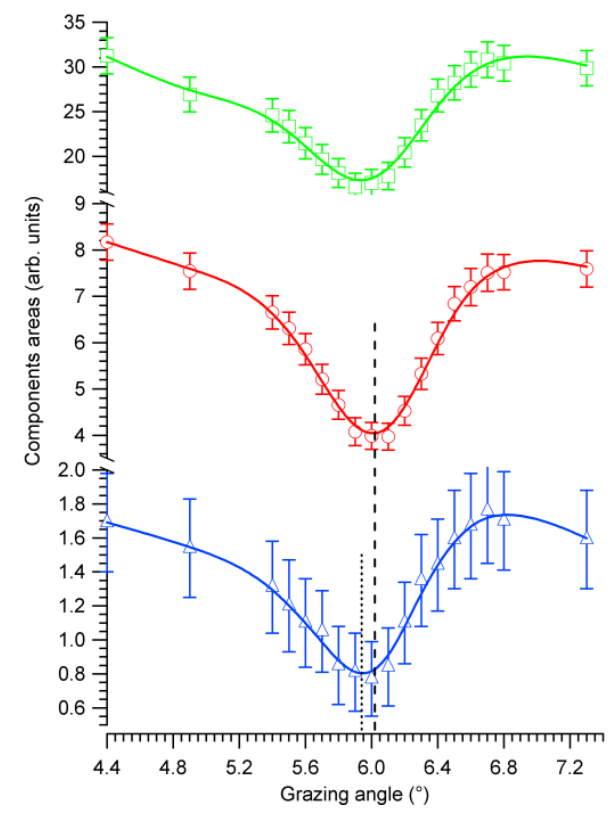

Figure 8: area of photoemission peaks (from top): carbon, $\mathrm{Ru} 3 \mathrm{~d}$ in silicide, and $\mathrm{Ru} 3 \mathrm{~d}$ not reacted vs rocking grazing angle.

In Figure 8 the areas of photoemission peaks of $\mathrm{C} 1 \mathrm{~s}$, of $\mathrm{Ru} 3 \mathrm{~d}$ in silicide, and of not reacted Ru 3d (from top to bottom) are reported. We stress the overall qualitative agreement with the results of the model calculation of the previous section. Moreover it is possible to observe that the two minima of $\mathrm{Ru}$ peaks are shifted, allowing a rough estimation of the interface extension of about $\sigma \sim 1 \mathrm{~nm}$ (compare the distance of the minima in Figure 8 with the minima in Figure 5 (left)).

\section{CONCLUSIONS}

A technique for the study of buried interfaces in multilayer was presented. The technique is based on the measures of the modulation of intensity of the photoemission peaks while scanning the rocking curve through the Bragg peak. This method was applied to the study of the buried $\mathrm{Ru} / \mathrm{Si}$ interface of a Ru capped $\left[\mathrm{Si}_{41.2} \mathrm{Mo}_{39.6}\right] \times 40$ multilayer stemming from the photoemission from $\mathrm{Ru} 3 \mathrm{~d}$ core levels. The advantage of photoemission is related with the sensitivity both to chemical species and chemical shifts due to compound formation.

Other interfaces have been studied including $\mathrm{Mo} / \mathrm{Si}$ and $\mathrm{Cr} / \mathrm{sc}$ in the corresponding multilayer structures. Results will be presented elsewhere.

The comparison of the model calculation with the experimental data indicates a sensitivity of the model to concentration profiles at the interfaces with possible sensitivity to the gradation of interface compounds.

One possible extension, from the experimental point of view, is to use the same pattern of excitation but different kind of emission (fluorescence).

\section{REFERENCES}

[1] M.J. Bedzik, G.M. Bommarito, J.S. Schildkraut, "X-ray standing waves at a reflecting mirror surface”, Phys. Rev. Lett. 62 (1989), 1376; L.G. Parrat, "Surface Studies of Solids by Total Reflection of X-Rays”, Phys. Rev. 95 (1954), 359 
[2] S. Nannarone, F. Borgatti, A. DeLuisa, B.P. Doyle, G.C. Gazzadi, A. Giglia, P. Finetti, N. Mahne, L. Pasquali, M. Pedio, G. Selvaggi, G. Naletto, M.G. Pelizzo, G. Tondello, "The BEAR beamline at Elettra", AIP Conference Proceedings 705 (2004), 450

[3] C. Calandra, O. Bisi, G. Ottaviani, "Electronic properties of silicon-transition metal interface compounds", Surf. Sci. Rep. 4 (1985), 271

[4] L. Pasquali, N. Mahne, M. Montecchi, V. Mattarello, S. Nannarone, "Formation and distribution of compounds at the Ru-Si(001) ultrathin film interface, Jour. App. Phys. 105 (2009), 044304

[5] D.G. Stearns, "The scattering of X-rays from nonideal multilayer structures", J. Appl. Phys. 65 (1989), 491

[6] A. Giglia, N. Mahne, F. Borgatti, B.P. Doyle, M. Pedio, S. Nannarone, "Spectroscopic diagnosis of the buried interface of $\mathrm{ML} \mathrm{Cr} / \mathrm{Sc}$ ", Poster presented to SILS, Napoli 2006

[7] S. Nannarone, A. Giglia, N. Mahne, A. DeLuisa, B.P. Doyle, F. Borgatti, M. Pedio, L. Pasquali, G. Naletto, M.G. Pelizzo, G. Tondello, "BEAR: a Bending Magnet for Emission Absorption and Reflectivity", NOTIZIARIO NEUTRONI E LUCE DI SINCROTRONE - Vol. 12, Pagg. 8-19 (2007)

[8] N.Mahne, L.Sponza, A.Verna, S.Nannarone, "OPAL (Optical Properties for Anisotropic Layers)" - to be published 